\title{
ASYMPTOTIC EXPANSION OF THE HETEROCLINIC BIFURCATION FOR THE PLANAR NORMAL FORM OF THE 1:2 RESONANCE
}

\author{
LUCI A. F. ROBERTO, PAULO R. DA SILVA, AND JOAN TORREGROSA
}

\begin{abstract}
We consider the family of planar differential systems depending on two real parameters

$$
\dot{x}=y, \quad \dot{y}=\delta_{1} x+\delta_{2} y+x^{3}-x^{2} y .
$$

This system corresponds to the normal form for the 1:2 resonance which exhibits a heteroclinic connection. The phase portrait of the system has a limit cycle which disappears in the heteroclinic connection for the parameter values on the curve $\delta_{2}=c\left(\delta_{1}\right)=-\frac{1}{5} \delta_{1}+O\left(\delta_{1}^{2}\right)$, $\delta_{1}<0$. We significantly improve the knowledge of this curve in a neighborhood of the origin.
\end{abstract}

\section{INTRODUCTION}

The theory of bifurcations is concerned to describe the variation of the qualitative behavior of the phase portrait when we vary the parameters. The bifurcation diagram consists of a partition of the parameter space such that, for parameters belonging to the same region, the respective phase portraits are topologically equivalent.

Consider a two-parameter family of differential systems in the plane. Suppose that there exists a curve such that for parameters values on this curve the systems exhibit heteroclinic connections. Moreover, suppose that when the parameters cross this curve we have the birth of an isolated periodic orbit, that is a limit cycle. In general, the existence of these curves is obtained by topological methods and very few properties are known about its asymptotic development.

For each $\sigma= \pm 1$, the differential system

$$
(\dot{x}, \dot{y})=\left(y, \delta_{1} x+\delta_{2} y+\sigma x^{3}-x^{2} y\right)
$$

is a versal deformation of a system with a double zero eigenvalue with a symmetry of order $2\left(\delta_{1}=\delta_{2}=0\right)$. These problems of codimension two were studied by Carr and Horozov in $[1,9]$. The unperturbed system is invariant under a rotation of the plane through an angle $2 \pi / q$ for $q=2$ and it can be proved that these are the normal forms for the 1:2 resonance, see $[2,10]$. The bifurcation diagram of this family is well known and a detailed analysis of them can be obtained in [10, Sect. 9.5.3] or [2, Sect. 4.2]. A heteroclinic connection appears for $\sigma=+1$. For $\sigma=-1$ a double homoclinic connection and a double limit cycle bifurcations occur. In particular the two limit cycles that emerge from the origin from a Hopf bifurcation disappear via a symmetric figure-eight homoclinic bifurcation.

In [7] the authors developed a method to obtain more accurate approximations of bifurcation curves corresponding to homoclinic and heteroclinic connections. Essentially, they seek polynomial approximations of the saddle separatrices and evaluate the contact of the

2010 Mathematics Subject Classification. Primary 34C23, 34C25, 34C37, 37C27.

Key words and phrases. Homoclinic Connections, Planar Systems, Bifurcation Diagram. 
vector field $(\dot{x}, \dot{y})$ with these algebraic curves. From this analysis it is possible to decide about the relative position of the saddle separatrix and thus determine if the parameters are above or below the bifurcation curve. Other related works where this technique is also applied are $[4,5,6,8]$. In the first two the authors improve the special bifurcation value in two 1-parameter families. In the third the Bogdanov-Takens bifurcation curve is studied globally in the 2-dimensional parameter space. The last deals with explicit upper and lower bounds for traveling waves. Another approach where a local study of a limit cycle bifurcation curve is done can be found in [11].

Here we restrict our attention to the case which exhibits a heteroclinic orbit connecting two symmetric saddles $s_{1}$ and $s_{2}(\sigma=1)$

$$
(\dot{x}, \dot{y})=\left(y, \delta_{1} x+\delta_{2} y+x^{3}-x^{2} y\right) .
$$

As far as we know, only the linear term of the heteroclinic bifurcation curve of the above system is done:

$$
C=\left\{\left(\delta_{1}, \delta_{2}\right), \delta_{2}=c\left(\delta_{1}\right)=-\frac{1}{5} \delta_{1}+O\left(\delta_{1}^{2}\right), \delta_{1}<0\right\} .
$$

In this paper, we apply the method developed in [7] to improve the knowledge on the heteroclinic bifurcation curve up to order 6 in a small neighborhood of the origin in the parameter space.

In order to clarify the bifurcation phenomenon we detail the results of $[1,9]$ in Section 2 adding an analysis of the global phase portrait on the Poincaré disc. The bifurcation diagram of (1) is presented in Figure 1.

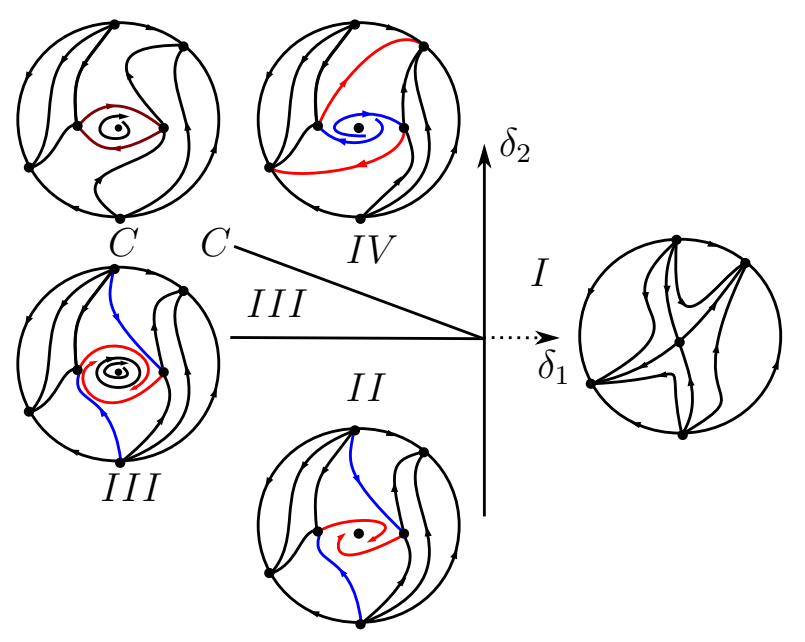

Figure 1. Phase portraits and bifurcation diagram for system (1).

The first result provides a good knowledge of the bifurcation curve for a small enough neighborhood of the origin. The second one explicitly gives an interval in which we have polynomial upper and lower bounds.

Theorem 1.1. Consider the 2-parameter family of differential systems given by (1). For every $d, D$ with $d<d_{6}<D$, there exists $\delta>0$ such that for $-\delta<\delta_{1}<0$ the heteroclinic 
bifurcation curve $\delta_{2}=c\left(\delta_{1}\right)$ satisfies

$$
\sum_{i=1}^{5} d_{i} \delta_{1}^{i}+d \delta_{1}^{6} \leq c\left(\delta_{1}\right) \leq \sum_{i=1}^{5} d_{i} \delta_{1}^{i}+D \delta_{1}^{6},
$$

with $d_{1}=-\frac{1}{5}, d_{2}=\frac{16}{13125}, d_{3}=\frac{5536}{103359375}, d_{4}=\frac{26032288}{8953505859375}, d_{5}=\frac{355355722144}{2016553357177734375}$, and $d_{6}=\frac{43256286929749984}{3784818582252960205078125}$.

For the best of our knowledge the analyticity of the bifurcation curve $c\left(\delta_{1}\right)$ is not proved. If it was true, then the coefficient of degree 6 of its Taylor expansion would be $d_{6}$. The necessary computations for our proof, as we will show in Section 3, are done with an algebraic manipulator ${ }^{1}$. For higher order terms the memory of our computer was not sufficient.

Theorem 1.2. Consider the 2-parameter family of differential systems given by (1). For every $\delta_{1} \in[-36 / 10201,0]$, the bifurcation curve $c\left(\delta_{1}\right)$ satisfies

$$
\left(N_{d} / D_{d}\right)\left(\sqrt{-\delta_{1}}\right)<c\left(\delta_{1}\right)<\left(N_{u} / D_{u}\right)\left(\sqrt{-\delta_{1}}\right),
$$

where

$$
\begin{aligned}
& N_{d}(\delta)=-6890625000 \sqrt{2} \delta^{2}-2756250000 \delta^{3}-317625000 \sqrt{2} \delta^{4} \\
&+50925000 \delta^{5}+1496250 \sqrt{2} \delta^{6}-2528200 \delta^{7}+19360 \sqrt{2} \delta^{8}+14641 \delta^{9}, \\
& D_{d}(\delta)= 131250\left(-262500 \sqrt{2}-105000 \delta-10500 \sqrt{2} \delta^{2}+160 \delta^{3}+121 \sqrt{2} \delta^{4}\right), \\
& N_{u}(\delta)=-6890625000 \sqrt{2} \delta^{2}-2756250000 \delta^{3}-317625000 \sqrt{2} \delta^{4} \\
& \\
&-76125000 \delta^{5}+7848750 \sqrt{2} \delta^{6}-7610200 \delta^{7}+58080 \sqrt{2} \delta^{8}+131769 \delta^{9}, \\
& D_{u}(\delta)=131250\left(-262500 \sqrt{2}-105000 \delta-10500 \sqrt{2} \delta^{2}+160 \delta^{3}+363 \sqrt{2} \delta^{4}\right) .
\end{aligned}
$$

We remark that the first terms of the series expansion of the functions $\left(N_{d} / D_{d}\right)(\delta)$ and $\left(N_{u} / D_{u}\right)(\delta)$ given in the above result coincide. More precisely

$$
\left(N_{u} / D_{u}-N_{d} / D_{d}\right)(\delta)=\frac{121 \sqrt{2}}{65625} \delta^{5}+O\left(\delta^{6}\right) .
$$

The series expansion up to order 5 of the function $c\left(\delta_{1}\right)$ in Theorem 1.1 together with the lower and upper bounds in Theorem 1.2 are drawn in Figure 2.

The paper is organized as follows. In Section 2 we describe the bifurcation diagram and the global phase portraits of system (1). In Sections 3 and 4 we prove the main results. The proofs are done obtaining the successive approximations of the stable and unstable invariant manifolds of the saddle equilibria.

\section{Global dynamics of system (1) on the Poincaré disk}

Consider the 2-parameter family of the planar differential systems (1). We remark that this system is invariant under the rotation through the angle $\pi$, that is $(x, y) \mapsto(-x,-y)$. Moreover it always has the equilibrium point $s_{0}=(0,0)$. Two other equilibria are $s_{1,2}=$

\footnotetext{
${ }^{1}$ The computations are done with MAPLE 18 in a Xeon computer (CPU E5-450, 3.0 GHz, RAM $32 \mathrm{~Gb}$ ) with GNU Linux.
} 


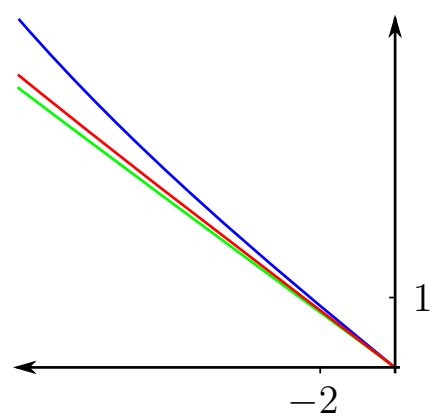

FiguRE 2. The green and blue curves are the lower and upper bounds in Theorem 1.2 and the red curve is the asymptotic expansion of the bifurcation curve in Theorem 1.1.

$\left(\mp \sqrt{-\delta_{1}}, 0\right)$ and bifurcate simultaneously from the trivial one via a pitchfork bifurcation along the line $F=\left\{\delta_{1}=0\right\}$. The nontrivial equilibria only exist for $\delta_{1}<0$.

In region $I=\left\{\delta_{1} \geq 0\right\}$ there is a single trivial equilibrium point $s_{0}$, which is a saddle. Crossing the lower branch of $F$ a pitchfork bifurcation is done generating a pair of symmetriccoupled saddles $s_{1,2}$, while the trivial equilibrium point becomes a stable node. This node turns into a focus somewhere in region $I I=\left\{\delta_{1}<0, \delta_{2} \leq 0\right\}$ and then loses its stability upon crossing the half-line $H=\left\{\delta_{1}<0, \delta_{2}=0\right\}$ via a nondegenerate Hopf bifurcation. In region $I I I=\left\{\delta_{1}<0,0<\delta_{2}<c\left(\delta_{1}\right)\right\}$ a unique and stable limit cycle exists. Crossing the curve $C$, given by (2), leads to the disappearance of the cycle through a heteroclinic bifurcation. Due to the symmetry, the heteroclinic orbits connecting the saddles $s_{1}$ and $s_{2}$ appear simultaneously, forming a heteroclinic cycle upon crossing $C$. In region $I V=$ $\left\{\delta_{1}<0, c\left(\delta_{1}\right)<\delta_{2}\right\}$ the totally unstable trivial equilibrium $D_{0}$ coexists with the saddles $s_{1,2}$. All three of these equilibria merge at the upper branch of the pitchfork bifurcation line $F$ as we return to region $I$. A more detailed analysis can be found in [10]. In the following proposition we summarize the properties of the phase portrait of system (1) in the Poincaré disk. See the bifurcation diagram and the phase portraits in Figure 1.

Proposition 2.1. The phase portrait of system (1) satisfies the following properties.

(a) On the infinity $S^{1}=\left\{(x, y): x^{2}+y^{2}=1\right\}$ system (1) does not depend on the parameters $\delta_{1}, \delta_{2}$. There exist four equilibrium points: two stable $\theta_{1}$ and $\theta_{3}$, corresponding to the directions $\theta=\pi / 4$ and $\theta=5 \pi / 4$, and two unstable $\theta_{2}$ and $\theta_{4}$, corresponding to the directions $\theta=\pi / 2$ and $\theta=3 \pi / 2$.

(b) For the parameter values on region $I$, the stable separatrices connect the saddle and the point $\theta_{2}$ and $\theta_{4}$. The unstable separatrices connect the saddle and $\theta_{1}$ and $\theta_{3}$.

(c) For the parameter values on region II, the stable separatrices of $s_{1}$ connect $s_{1}$ and $\theta_{2}$ and $s_{1}$ and $\theta_{4}$. The unstable separatrices of $s_{1}$ connect $s_{1}$ and $s_{0}$ and $s_{1}$ and $\theta_{3}$. The stable separatrices of $s_{2}$ connect $s_{2}$ and $\theta_{2}$ and $s_{2}$ and $\theta_{4}$. The unstable separatrix of $s_{2}$ connects $s_{2}$ and $s_{0}$ and $s_{2}$ and $\theta_{1}$.

(d) For the parameter values on region III, the stable separatrices of $s_{1}$ connect $s_{1}$ and the equilibrium on the infinity $\theta_{2}$ and $\theta_{4}$. The stable separatrices of $s_{1}$ connect $s_{1}$ and $\theta_{2}$ and $s_{1}$ and $\theta_{4}$. The unstable separatrices of $s_{1}$ connect $s_{1}$ and the limit cycle and $s_{1}$ and $\theta_{3}$. The stable separatrices of $s_{2}$ connect $s_{2}$ and $\theta_{2}$ and $s_{2}$ and $\theta_{4}$. The unstable separatrix of $s_{2}$ connects $s_{2}$ and the limit cycle and $s_{2}$ and $\theta_{1}$. 
(e) For the parameter values on region $I V$, the stable separatrices of $s_{1}$ connect $s_{1}$ and $\theta_{2}$ and $s_{1}$ and $s_{2}$. The unstable separatrices of $s_{1}$ connect $s_{1}$ and $s_{2}$ and $s_{1}$ and $\theta_{3}$. The stable separatrices of $s_{2}$ connect $s_{2}$ and $s_{1}$ and $s_{2}$ and $\theta_{4}$. The unstable separatrix of $s_{2}$ connects $s_{2}$ and $s_{1}$ and $s_{2}$ and $\theta_{1}$.

(f) For the parameter values on region $V$, the stable separatrices of $s_{1}$ connect $s_{1}$ and $\theta_{2}$ and $s_{1}$ and $s_{0}$. The unstable separatrices of $s_{1}$ connect $s_{1}$ and $\theta_{1}$ and $s_{1}$ and $\theta_{3}$. The stable separatrices of $s_{2}$ connect $s_{2}$ and $s_{0}$ and $s_{2}$ and $\theta_{4}$. The unstable separatrix of $s_{2}$ connects $s_{2}$ and $\theta_{3}$ and $s_{2}$ and $\theta_{1}$.

Proof. Using the technique detailed in [3], we determine the phase portrait on the infinity analyzing the system

$$
\dot{\theta}=v^{3}(\dot{y} \cos \theta-\dot{x} \sin \theta), \quad x=\frac{\cos \theta}{v}, \quad y=\frac{\sin \theta}{v}, \quad v=0 .
$$

It does not depend neither $\delta_{1}$ nor $\delta_{2}$. In fact, equation (3) for system (1) is

$$
\dot{\theta}=\cos ^{4} \theta-\cos ^{3} \theta \sin \theta \text {. }
$$

Thus the equilibrium points on the infinity correspond to the directions $\theta=\pi / 4, \pi / 2,5 \pi / 4$ and $3 \pi / 2$. The stability of each equilibrium is determined by the sign of $\dot{\theta}$. The complete picture can be obtained using the $\mathrm{P} 4$ program, [3], for example. The statements follow directly from the local analysis, the symmetry and Poincaré-Bendixon Theorem.

\section{SMALl Neighborhood of the ORIGin IN THE PARAMETERS SPACE}

This section is devoted to the proof of Theorem 1.1. It follows from two technical results. In the first one we study the relative position of the parameter values $\left(\delta_{1}, \delta_{2}\right)$ with respect to the bifurcation curve from the existence of curves without contact. In the second one, after a change of variables in the parameter space, we provide the lower and upper bounds for the heteroclinic bifurcation curve. From now on, the vector field associated to (1) is denoted by

$$
(P(x, y), Q(x, y))=\left(y, \delta_{1} x+\delta_{2} y+x^{3}-x^{2} y\right) .
$$

Lemma 3.1. Consider the 2-parameter family of vector fields given by (4) and denote by $I^{-}=\left(-\sqrt{-\delta_{1}}, 0\right]$ and $I^{+}=\left[0, \sqrt{-\delta_{1}}\right)$. Suppose that $\left(\delta_{1}, \delta_{2}\right)$ are parameter values satisfying that:

(a) there exist polynomials $p^{ \pm}(x)$ satisfying that $p^{ \pm}(x)>0$ in $I^{ \pm}$and $p^{ \pm}\left( \pm \sqrt{-\delta_{1}}\right)=0$;

(b) denoting by $f^{ \pm}(x, y)=y-p^{ \pm}(x)$, then $\nabla f^{ \pm} \cdot(P, Q)<0$ (resp. $>0$ ) on the points $\left(x, p^{ \pm}(x)\right), x \in I^{ \pm}$and

(c) $p^{-}(0)-p^{+}(0)<0$ (resp. $\left.>0\right)$.

Then the bifurcation curve $(2)$ satisfies $c\left(\delta_{1}\right)>\delta_{2}$ (resp. $\left.c\left(\delta_{1}\right)<\delta_{2}\right)$.

Proof. The hypotheses imply that the curves $f^{-}(x, y)=0$ and $f^{+}(x, y)=0$ pass through the points $s_{1}$ and $s_{2}$ defined in Section 2, and the graph of $f^{-}(x, y)=0$ intersects the $y$-axis down the graph of $f^{+}(x, y)=0$. Furthermore (b) and (c) imply that the unstable separatrix of $s_{1}$ for $x \in I^{-}$is under the curve $f^{-}(x, y)=0$ and the unstable separatrix of $s_{2}$ for $x \in I^{+}$is over the curve $f^{+}(x, y)=0$. Thus, the phase portrait corresponding to this configuration is the one given in region III, see Figures 1 and 3. Shortly, $\delta_{2}<c\left(\delta_{1}\right)$. The other configuration can be proved similarly. 

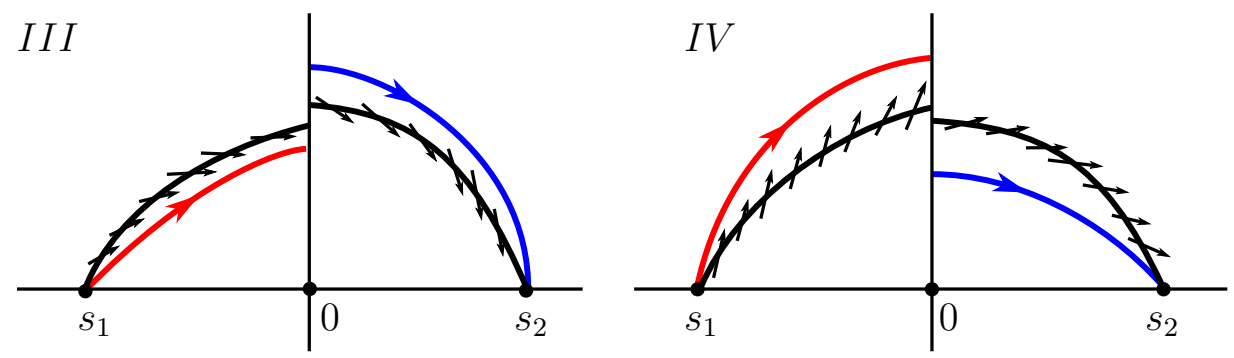

FIGURE 3. Relative position of the saddle separatrices with respect to the algebraic curves $y=p^{+}(x)$ and $y=p^{-}(x)$.

Lemma 3.2. Consider the 2-parameter family of vector fields given by (4) with

$$
\delta_{1}=-\varepsilon_{1}^{2}, \quad \delta_{2}=\frac{\varepsilon_{1}^{4}+8 \varepsilon_{1}^{2}-\varepsilon_{2}^{2}}{2 \varepsilon_{1}^{2}+2 \varepsilon_{2}} .
$$

With these new parameters the bifurcation curve $c\left(\delta_{1}\right)$ defined in $(2)$ changes to $\widetilde{c}\left(\varepsilon_{1}\right)$. For every $A<\frac{43256286929749984}{3784818582252960205078125}<B$, there exists $\varepsilon_{0}>0$ such that

$$
-b_{1} \varepsilon_{1}-b_{2} \varepsilon_{1}^{2}-\ldots-b_{11} \varepsilon_{1}^{11}-B \varepsilon_{1}^{12}<\widetilde{c}\left(\varepsilon_{1}\right)<-b_{1} \varepsilon_{1}-b_{2} \varepsilon_{1}^{2}-\ldots-b_{11} \varepsilon_{1}^{11}-A \varepsilon_{1}^{12},
$$

for $0 \leq \varepsilon_{1}<\varepsilon_{0}$ with

$$
\begin{aligned}
b_{1} & =-2 \sqrt{2}, & b_{5} & =\frac{121}{65625} \sqrt{2}, & b_{9} & =\frac{34623151}{7162804687500} \sqrt{2}, \\
b_{2} & =\frac{1}{5}, & b_{6} & =-\frac{5536}{103359375}, & b_{10} & =-\frac{355355722144}{2016553357177734375}, \\
b_{3} & =-\frac{2}{25} \sqrt{2}, & b_{7} & =-\frac{43747}{516796875} \sqrt{2}, & b_{11} & =-\frac{62085189031889}{201655335717773437500} \sqrt{2} . \\
b_{4} & =\frac{16}{13125}, & b_{8} & =\frac{26032288}{8953505859375}, & &
\end{aligned}
$$

Proof of Lemma 3.2. We consider the vector field (4) under the change of parameters given by $(5)$. With these parameters the saddle points are located in $\left(-\varepsilon_{1}, 0\right)$ and $\left(\varepsilon_{1}, 0\right)$. Since they are hyperbolic, the Hartman-Grobman Theorem guaranties the existence of stable and unstable curves tangent to the stable and unstable eigenspaces respectively. The slopes of the eigenvectors are

$$
a_{1}^{+}=-\frac{\varepsilon_{1}^{2}}{2}-\frac{\varepsilon_{2}}{2}, \quad a_{1}^{-}=\frac{4 \varepsilon_{1}^{2}}{\varepsilon_{1}^{2}+\varepsilon_{2}} .
$$

We start describing the procedure for determining a degree $k$ polynomial approximation of the function $\widetilde{c}\left(\varepsilon_{1}\right)$ in a neighborhood of the origin

$$
\varepsilon_{2}=\widetilde{c}\left(\varepsilon_{1}\right)=-b_{1} \varepsilon_{1}-b_{2} \varepsilon_{1}^{2}-\ldots-b_{k-1} \varepsilon_{1}^{k-1}-b_{k} \varepsilon_{1}^{k} .
$$

Firstly we obtain, determining the coefficients $a_{i}^{ \pm}$, the higher degree polynomial approximations of that stable and unstable curves

$$
y_{k+1}^{ \pm}=\sum_{i=1}^{k+1} a_{i}^{ \pm}\left(x \mp \varepsilon_{1}\right)^{i},
$$


imposing that $y_{k+1}^{ \pm}$are solutions of the differential equation (1) until order $k+1$. Because of the huge size of the coefficients $a_{i}^{ \pm}$here we only show the first

$$
a_{2}^{+}=-\frac{\varepsilon_{1}\left(\varepsilon_{1}^{2}+\varepsilon_{2}+3\right)\left(\varepsilon_{1}^{2}+\varepsilon_{2}\right)}{\varepsilon_{1}^{4}+4 \varepsilon_{1}^{2}+\varepsilon_{2}^{2}+2 \varepsilon_{2} \varepsilon_{1}^{2}}, \quad a_{2}^{-}=\frac{2 \varepsilon_{1}\left(5 \varepsilon_{1}^{2}-3 \varepsilon_{2}\right)}{\varepsilon_{1}^{4}+16 \varepsilon_{1}^{2}+\varepsilon_{2}^{2}+2 \varepsilon_{2} \varepsilon_{1}^{2}} .
$$

Secondly we consider $x=\varepsilon_{1}-z$ in the case $(+)$ and $x=-\varepsilon_{1}+z$ in the case $(-)$. Since $0 \leq x<\varepsilon_{1}$ and $-\varepsilon_{1}<x \leq 0$, respectively, then $0 \leq z<\varepsilon_{1}$.

Now we compute the following functions

$$
\begin{aligned}
w_{k+1}^{ \pm}\left(\varepsilon_{1}, b, z\right) & =\left(-\frac{d y_{k+1}^{ \pm}}{d x}, 1\right) \cdot(P, Q), \\
\Delta_{k+1}\left(\varepsilon_{1}, b\right) & =y_{k+1}^{-}(0)-y_{k+1}^{+}(0),
\end{aligned}
$$

using (8). The functions $w_{k+1}^{ \pm}\left(\varepsilon_{1}, b, z\right)$ are rational functions with strictly positive denominators

$$
w_{k+1}^{ \pm}\left(\varepsilon_{1}, b, z\right)=\frac{z^{k+2} \alpha_{k+1}^{ \pm}\left(\varepsilon_{1}, b, z\right)}{g_{k+1}^{ \pm}\left(\varepsilon_{1}, b\right)^{2}} .
$$

In a neighborhood of $z=0$ the principal term of the series of $w_{k+1}^{ \pm}$in $z$ is determined by $\alpha_{k+1}^{ \pm}\left(\varepsilon_{1}, b, 0\right)$. Moreover, the series expansion of $\alpha_{k+1}^{ \pm}\left(\varepsilon_{1}, b\right)$ and $\Delta_{k+1}\left(\varepsilon_{1}, b, z\right)$ in $\varepsilon_{1}$ write as

$$
\begin{aligned}
\alpha_{k+1}^{+}\left(\varepsilon_{1}, b, 0\right) & =\gamma_{k}^{+}(b) \varepsilon_{1}^{m_{k}}+O\left(\varepsilon_{1}^{m_{k}+1}\right), \\
\alpha_{k+1}^{-}\left(\varepsilon_{1}, b, 0\right) & =\gamma_{k}^{-}(b) \varepsilon_{1}^{n_{k}}+O\left(\varepsilon_{1}^{n_{k}+1}\right), \\
\Delta_{k+1}\left(\varepsilon_{1}, b\right) & =\gamma_{k}^{0}(b) \varepsilon_{1}^{k+1}+O\left(\varepsilon_{1}^{k+2}\right) .
\end{aligned}
$$

For $k=1$ we obtain that

$$
\begin{aligned}
& \gamma_{1}^{+}(b)=-2\left(b^{2}-2\right)\left(b^{2}-8\right) \\
& \gamma_{1}^{-}(b)=-b\left(b^{2}-32\right)\left(b^{2}-8\right) \\
& \gamma_{1}^{0}(b)=\frac{1}{2} \frac{\left(b^{2}-8\right)\left(b^{4}+26 b^{2}+64\right)}{b\left(b^{2}+16\right)\left(b^{2}+4\right)}
\end{aligned}
$$

with $m_{1}=4$ and $n_{1}=5$. Vanishing $\gamma_{1}^{ \pm}(b)$ and $\gamma_{1}^{0}(b)$ simultaneously we have that $b_{1}= \pm 2 \sqrt{2}$.

For $k=2, \ldots, 11$, fixing for example $b_{1}=-2 \sqrt{2}$, we obtain that $m_{k}=n_{k}, \gamma_{k}^{ \pm}(b)=$ $c_{k}^{ \pm}\left(b-b_{k}\right)$, and $\gamma_{k}^{0}(b)=c_{k}^{0}\left(b-b_{k}\right)$ with $c_{k}^{ \pm}$and $c_{k}^{0}$ nonzero algebraic numbers. Hence the expressions of the coefficients $b_{k}$ given in (6) can be obtained recurrently vanishing also $\gamma_{k}^{ \pm}(b)$ and $\gamma_{k}^{0}(b)$.

For $k=12$ we have that $m_{12}=n_{12}=140, \gamma_{12}^{ \pm}(b)=c_{12}^{ \pm}\left(b-b_{12}\right)$, and $\gamma_{12}^{0}(b)=c_{12}^{0}\left(b-b_{12}\right)$ with

$$
c_{12}^{+}=2^{190} \cdot 3^{36} \cdot 5^{11} \cdot 7^{6} \cdot 11^{2} \cdot 13^{2} \cdot \sqrt{2}, \quad c_{12}^{-}=2^{56} c_{12}^{+}, \quad c_{12}^{0}=43 \cdot 127 \cdot 2^{-12} .
$$

We have written $c_{12}^{0}$ and $c_{12}^{ \pm}$using their decomposition in powers of prime numbers because of the size of them. For $\varepsilon_{1}$ small enough, as the numbers $c_{12}^{ \pm}$and $c_{12}^{0}$ are positive, the signs of $\alpha_{k+1}^{+}\left(\varepsilon_{1}, b, 0\right), \alpha_{k+1}^{-}\left(\varepsilon_{1}, b, 0\right)$, and $\Delta_{k+1}\left(\varepsilon_{1}, b\right)$ are determined by $\left(b-b_{12}\right)$. Hence the lower bound and the upper bound given in the statement follow applying Lemma 3.1.

Proof of Theorem 1.1. Using Lemma 3.2 and coming back to the original parameters we conclude the proof. We remark that the coefficients $d_{k}$ in the statement are related with the coefficients $b_{k}$ from Lemma 3.2 in the following way: $d_{k}=(-1)^{k} b_{2 k}$ for $k=1, \ldots, 6$. 
In what follows we explain why we consider this change of coordinates. Consider the 2-parameter $\left(\delta_{1}, \delta_{2}\right)$ family of differential systems given by (1). First of all we consider the change of the parameters given by $\delta_{1}=-\varepsilon_{1}^{2}$. Thus the saddle points become $\left(-\varepsilon_{1}, 0\right)$ and $\left(\varepsilon_{1}, 0\right)$ and it will facilitate next computations. With these new parameters the vector field associated to (1) is given by $(P(x, y), Q(x, y))=\left(y,-\varepsilon_{1}^{2} x+\delta_{2} y+x^{3}-x^{2} y\right)$.

In the proof of Lemma 3.2 we write the linear approximation of the stable and unstable separatrices of the saddle point $\left(\varepsilon_{1}, 0\right)$ as $y=a_{1}^{ \pm}\left(x-\varepsilon_{1}\right)+O\left(\left(x-\varepsilon_{1}\right)^{2}\right)$. Straightforward computations show that

$$
a_{1}^{ \pm}=\frac{\delta_{2}}{2}-\frac{\varepsilon_{1}^{2}}{2} \mp \frac{\sqrt{\delta_{2}^{2}-2 \delta_{2} \varepsilon_{1}^{2}+\varepsilon_{1}^{4}+8 \varepsilon_{1}^{2}}}{2} .
$$

The same expressions are also valid for the saddle point $\left(-\varepsilon_{1}, 0\right)$. The computations are easier if we eliminate all the expressions involving square roots. The proposed change of variables (5) transforms the above expressions in the two rational ones given in (7).

Now we comment the main difficulties to go further in the development of the function $c\left(\delta_{1}\right)$ given in Theorem 1.1. The key point in the proof of Lemma 3.2 is the explicit computation of $\gamma_{k}^{ \pm}(b) \varepsilon_{1}^{m_{k}}$ and $\gamma_{k}^{0}(b) \varepsilon_{1}^{k+1}$ in (11). Although we have obtained $\gamma_{k}^{ \pm}(b) \varepsilon_{1}^{m_{k}}$ for $k=13, \ldots, 25$ the computation of $\gamma_{13}^{0}(b) \varepsilon_{1}^{14}$ exceed the memory of our computer ${ }^{2}$. From these $\gamma_{k}(b)^{ \pm}$we can obtain the following values

$$
\begin{aligned}
d_{7} & =\frac{168679322468558257992224}{217371120122920355358123779296875}, \\
d_{8} & =\frac{4305872427368562628639420153568}{79066218005440850312386486530303955078125}, \\
d_{9} & =\frac{37564005983311734369133435252462168672}{9586472551564932017081900994164049625396728515625}, \\
d_{10} & =\frac{2766766190160174565678452208821237437436993056}{9624031536171796537705606430297599867884814739227294921875}, \\
d_{11} & =\frac{8624760946313011664012747865814391868142139840873664416}{9624031536171796537705606430297599867884814739227294921875 ;}, \\
d_{12} & =\frac{5446438458418037504482627999300013311390638864395503052380413344}{3^{21} \cdot 5^{33} \cdot 7^{11} \cdot 11^{9} \cdot 13^{8} \cdot 17^{6} \cdot 19^{5} \cdot 23^{3}} .
\end{aligned}
$$

However to prove that these values provide the expansion up to order 12 in $\delta_{1}$ we need to know $\gamma_{k}^{0}(b)$ for $k \geq 13$.

\footnotetext{
${ }^{2}$ Intel Xeon, 2.6GHz, 32Gb RAM.
} 
Finally, we remark that, as in [7], the prime decompositions of the denominators of $d_{k}$ for $k=1, \ldots, 12$ have a nice and regular structure:

$$
\begin{aligned}
& 5, \\
& 3 \cdot 5^{4} \cdot 7, \\
& 3^{3} \cdot 5^{7} \cdot 7^{2}, \\
& 3^{5} \cdot 5^{10} \cdot 7^{3} \cdot 11, \\
& 3^{7} \cdot 5^{12} \cdot 7^{4} \cdot 11^{2} \cdot 13, \\
& 3^{8} \cdot 5^{16} \cdot 7^{5} \cdot 11^{3} \cdot 13^{2}, \\
& 3^{11} \cdot 5^{19} \cdot 7^{6} \cdot 11^{4} \cdot 13^{3} \cdot 17, \\
& 3^{13} \cdot 5^{22} \cdot 7^{7} \cdot 11^{5} \cdot 13^{4} \cdot 17^{2} \cdot 19, \\
& 3^{14} \cdot 5^{25} \cdot 7^{8} \cdot 11^{6} \cdot 13^{5} \cdot 17^{3} \cdot 19^{2}, \\
& 3^{17} \cdot 5^{26} \cdot 7^{9} \cdot 11^{7} \cdot 13^{6} \cdot 17^{4} \cdot 19^{3} \cdot 23, \\
& 3^{19} \cdot 5^{30} \cdot 7^{10} \cdot 11^{8} \cdot 13^{7} \cdot 17^{5} \cdot 19^{4} \cdot 23^{2}, \\
& 3^{21} \cdot 5^{33} \cdot 7^{11} \cdot 11^{9} \cdot 13^{8} \cdot 17^{6} \cdot 19^{5} \cdot 23^{3} .
\end{aligned}
$$

where the small irregularity with the number of threes and fives could be produced by some cancellations with the respective numerators. Unfortunately no regularity appears in the numerators.

\section{FiXed NEIGHBORHOOD OF THE ORIGIN IN THE PARAMETERS SPACE}

We start this section with a proposition which is essentially the version of Theorem 1.2 using the parameters $\varepsilon_{1}, \varepsilon_{2}$ introduced in (5).

Proposition 4.1. Consider the 2-parameter family of vector fields given by (4) with the parameters given by (5). The heteroclinic bifurcation curve $\varepsilon_{2}=\widetilde{c}\left(\varepsilon_{1}\right)$ satisfies

$$
-b_{1} \varepsilon_{1}-\ldots-b_{4} \varepsilon_{1}^{4}-\frac{121}{43750} \sqrt{2} \varepsilon_{1}^{5}<\widetilde{c}\left(\varepsilon_{1}\right)<-b_{1} \varepsilon_{1}-\ldots-b_{4} \varepsilon_{1}^{4}-\frac{121}{131250} \sqrt{2} \varepsilon_{1}^{5}
$$

for any $\varepsilon_{1} \in[0,6 / 101]$, with coefficients $b_{i}$ given in equation (6).

Proof. We consider system vector field (1) with parameters given by (5). Taking $k=5$ in the proof of Lemma 3.2 we can see that, for $\varepsilon_{1}$ small enough, for every $A<\frac{121}{65625} \sqrt{2}<B$, there exists $\varepsilon_{0}>0$ such that

$$
-b_{1} \varepsilon_{1}-b_{2} \varepsilon_{1}^{2}-\ldots-b_{4} \varepsilon_{1}^{4}-B \varepsilon_{1}^{5}<\widetilde{c}\left(\varepsilon_{1}\right)<-b_{1} \varepsilon_{1}-b_{2} \varepsilon_{1}^{2}-\ldots-b_{5} \varepsilon_{1}^{5}-A \varepsilon_{1}^{5},
$$

for $0 \leq \varepsilon_{1}<\varepsilon_{0}$ with $b_{i}, i=1,2,3,4$ in (6), $m_{5}=n_{5}=40$,

$$
c_{5}^{+}=2^{50} \cdot 3^{11} \cdot 5^{2} \cdot 7^{2} \sqrt{2}, \quad c_{5}^{-}=2^{66} \cdot 3^{11} \cdot 5^{2} \cdot 7^{2} \sqrt{2}, \quad \text { and } c_{5}^{0}=\frac{127}{96} .
$$

Consequently, the functions $w_{6}^{ \pm}$and $\Delta_{6}$ defined in (9) satisfy

$$
\begin{array}{lll}
w_{6}^{+}\left(\varepsilon_{1}, A, z\right)<0, & w_{6}^{-}\left(\varepsilon_{1}, A, z\right)<0, & \Delta_{6}\left(\varepsilon_{1}, A\right)<0, \\
w_{6}^{+}\left(\varepsilon_{1}, B, z\right)>0, & w_{6}^{-}\left(\varepsilon_{1}, B, z\right)>0, & \Delta_{6}\left(\varepsilon_{1}, B\right)>0,
\end{array}
$$


in a neighborhood of $\left(\varepsilon_{1}, z\right)=(0,0)$. The proof follows from Lemma 3.1, taking $B=3 b_{5} / 2=$ $121 \sqrt{2} / 43750$ and $A=b_{5} / 2=121 \sqrt{2} / 131250$, proving that these signs do not change for $z \in\left[0, \varepsilon_{1}\right)$ and $\varepsilon_{1} \in[0,6 / 101]$.

According (10) the signs of $w_{6}^{ \pm}\left(\varepsilon_{1}, b, z\right)$ are determined by the signs of $\alpha_{6}^{ \pm}\left(\varepsilon_{1}, b, z\right)$ that are polynomials of degree 4 in $z$ and degree 190 in $\varepsilon_{1}$. Moreover, the function $\Delta_{6}$ is a given rational function. Consider, for $b=A$, the following eight polynomials

$$
\begin{array}{ll}
p_{1}\left(\varepsilon_{1}\right)=\alpha_{6}^{+}\left(\varepsilon_{1}, A, 0\right), & p_{5}\left(\varepsilon_{1}\right)=\alpha_{6}^{-}\left(\varepsilon_{1}, A, \varepsilon_{1}\right), \\
p_{2}\left(\varepsilon_{1}\right)=\alpha_{6}^{+}\left(\varepsilon_{1}, A, \varepsilon_{1}\right), & p_{6}\left(\varepsilon_{1}\right)=\operatorname{disc}\left(\alpha_{6}^{-}\left(\varepsilon_{1}, A, z\right), z\right), \\
p_{3}\left(\varepsilon_{1}\right)=\operatorname{disc}\left(\alpha_{6}^{+}\left(\varepsilon_{1}, A, z\right), z\right), & p_{7}\left(\varepsilon_{1}\right)=\operatorname{numer}\left(\Delta_{6}\left(\varepsilon_{1}, A\right)\right) / \varepsilon_{1}^{6}, \\
p_{4}\left(\varepsilon_{1}\right)=\alpha_{6}^{-}\left(\varepsilon_{1}, A, 0\right), & p_{8}\left(\varepsilon_{1}\right)=\operatorname{denom}\left(\Delta_{6}\left(\varepsilon_{1}, A\right)\right) .
\end{array}
$$

The degrees of them are 190, 190, 1088, 190, 190, 1088, 164 and 164, respectively. Here we have denoted by $\operatorname{disc}(\cdot, z)$ the discriminant function with respect to $z$. Using the Sturm method we can localize the small positive zero of all them and it is $\rho^{u} \approx 0.05944857762$. Thus we conclude that, for $b=A$, the prescribed signs of the functions $w_{6}^{ \pm}$and $\Delta_{6}$ remain unchanged for $z \in\left[0, \varepsilon_{1}\right]$ and $\varepsilon_{1}<\rho^{u}$. Arguing in a similar way, for $b=B$, we can also prove that $w_{6}^{+}\left(\varepsilon_{1}, B, z\right)>0, w_{6}^{-}\left(\varepsilon_{1}, B, z\right)>0$, and $\Delta_{6}\left(\varepsilon_{1}, B\right)>0$, for $z \in\left[0, \varepsilon_{1}\right]$ and $\varepsilon_{1}<\rho^{d}$ with $\rho^{d} \approx 0.3135863722$. Clearly the proof finishes because $6 / 101 \leq \min \left\{\rho^{d}, \rho^{u}\right\}$.

Proof of Theorem 1.2. The change of parameters (5) reverses the position of the upper and lower curves given in Proposition 4.1. With this change the interval $[0,6 / 101]$ for $\varepsilon_{1}$ moves to $[-36 / 10201,0]$ for $\delta_{1}$.

\section{ACKNOWLEDGEMENTS}

The first author is partially supported by CAPES and FAPESP. The second author is partially supported by CAPES, CNPq-Brazil, and FAPESP. The third author is partially supported by the MINECO/FEDER grants MTM2008-03437, MTM2013-40998-P, and UNAB13-4E-1604; the AGAUR grant 2014 SGR568. All authors are partially supported by FP7-PEOPLE-2012-IRSES 318999.

\section{REFERENCES}

[1] J. Carr. Applications of centre manifold theory, volume 35 of Applied Mathematical Sciences. SpringerVerlag, New York-Berlin, 1981.

[2] S.-N. Chow, C. Z. Li, and D. Wang. Normal forms and bifurcation of planar vector fields. Cambridge University Press, Cambridge, 1994.

[3] F. Dumortier, J. Llibre, and J. C. Artés. Qualitative theory of planar differential systems. Universitext. Springer-Verlag, Berlin, 2006.

[4] J. D. García-Saldaña, A. Gasull, and H. Giacomini. Bifurcation diagram and stability for a oneparameter family of planar vector fields. J. Math. Anal. Appl., 413(1):321-342, 2014.

[5] J. D. García-Saldaña, A. Gasull, and H. Giacomini. Bifurcation values for a family of planar vector fields of degree five. Discrete Contin. Dyn. Syst., 35(2):669-701, 2015.

[6] A. Gasull, H. Giacomini, S. Pérez-González, and J. Torregrosa. A proof of Perko's conjectures for the Bogdanov-Takens system. J. Differential Equations, 255(9):2655-2671, 2013.

[7] A. Gasull, H. Giacomini, and J. Torregrosa. Some results on homoclinic and heteroclinic connections in planar systems. Nonlinearity, 23(12):2977-3001, 2010.

[8] A. Gasull, H. Giacomini, and J. Torregrosa. Explicit upper and lower bounds for the traveling wave solutions of Fisher-Kolmogorov type equations. Discrete Contin. Dyn. Syst., 33(8):3567-3582, 2013. 
[9] E. I. Horozov. Versal deformations of equivariant vector fields for cases of symmetry of order 2 and 3. Trudy Sem. Petrovsk., (5):163-192, 1979.

[10] Y. A. Kuznetsov. Elements of applied bifurcation theory, volume 112 of Applied Mathematical Sciences. Springer-Verlag, New York, second edition, 1998.

[11] R. López-Ruiz and J. L. López. Bifurcation curves of limit cycles in some Liénard systems. Internat. J. Bifur. Chaos Appl. Sci. Engrg., 10(5):971-980, 2000.

Instituto de Biociências, Letras e Ciências Exatas, UnesP - Univ Estadual Paulista, Campus de São José do Rio Preto, Departamento de Matemática

E-mail address: lroberto@ibilce.unesp.br

Instituto de Biociências, Letras e Ciências Exatas, UneSP - Univ Estadual Paulista, Campus de São José do Rio Preto, Departamento de Matemática

E-mail address: prs@ibilce.unesp.br

Departament de Matemàtiques, Universitat Autònoma de Barcelona, 08193 Bellaterra, Barcelona, Catalonia, Spain

E-mail address: torre@mat.uab.cat 